\section{Matrix metalloproteinases: not-so-innocent bystanders in heart failure}

\author{
Richard T. Lee and Peter Libby
}

Commentary

See related article,

pages 857-866.

Cardiovascular Division, Department of Medicine, Brigham and Women's Hospital, Boston, Massachusetts, USA

Address for correspondence: Richard T. Lee, Cardiovascular Division, Department of Medicine, Brigham and Women's Hospital,

75 Francis Street, Boston, Massachusetts 02115, USA. Phone: (617) 732-7146; Fax: (617) 264-5139; E-mail: rlee@rics.bwh.harvard.edu.

For decades, the classic teaching of the pathophysiology of heart failure has focused, quite reasonably, on the inside of the cardiac myocyte. The dynamic states of the contractile proteins and exquisite controls of intracellular calcium movements coordinate myocardial contraction and relaxation and appeared to be prime suspects in all forms of systolic and diastolic dysfunction. Furthermore, molecular genetics has implicated mutations in the cytoskeleton as important causes of cardiomyopathy in both mice and humans (1). In contrast, the extracellular matrix (ECM) seemed to serve only a passive role, providing a relatively static external structure whose rates of metabolic turnover appeared much too slow to influence cardiac contractility or hypertrophy directly. Cardiac myocytes did their work on the inside, while the matrix seemed to just hang on for the ride.

It is not surprising, then, that the potential role in heart failure has only recently emerged for the matrix metalloproteinases (MMPs), a family of enzymes with broad roles in ECM metabolism. While processes such as inflammatory destruction of articular cartilage matrix or invasion of metastatic cancer cells clearly depend upon active ECM degradation, the role of ECM degradation in myocardial hypertrophy and dysfunction is less intuitive. MMPs are overexpressed in many forms of myocardial dysfunction in both experimental models and human diseases (2), but MMP overexpression is ubiquitous in changing or remodeling tissues. Thus, the enzymes could easily be taken for innocent bystanders in heart failure.

For several reasons, MMPs must now be regarded as viable suspects in heart failure. First, the ECM is both physically and biochemically in close communication with the cytoskeleton. The general concept that matrix molecules can provide powerful "outside-in" cel- lular signals through ECM receptors such as $\beta 1$ integrins applies to the cardiac myocyte (3). Furthermore, molecular defects in the dystrophin-dystroglycan-laminin complex, which links the cytoskeleton with the ECM, have been shown to cause cardiomyopathy in both humans and animals (4). In addition, studies of MMP inhibitors in different animal models $(5,6)$ as well as in transgenic mice with deletion of MMP9 (7) demonstrate that MMPs can profoundly influence the process of cardiac dilation, a central feature of heart failure progression.

In this issue of the JCI, Kim et al. (8) further implicate MMPs in heart failure by providing direct evidence that disruption of the ECM by MMP-1 can cause cardiac hypertrophy and dysfunction. By overexpressing human MMP-1, a collagenase that initiates degradation of fibrillar collagen, in cardiac ventricles of mice, they have developed a unique model for demonstrating the importance of the collagen scaffold of the myocardium. By 6 months of age, these animals develop myocyte hypertrophy, and by 12 months they develop both systolic and diastolic dysfunction. Their experimental model is particularly interesting because mice and other rodents carry no endogenous $M M P-1$ gene; another metalloproteinase, MMP-13, appears to serve as a fibrillar collagenase in these species. Thus, the experiment was not confounded by compensatory changes in expression of a mouse MMP-1 homologue.

This study is also of interest for what it does not show. Deletion of MMPs in genetically engineered mice has resulted in predominantly mild or normal phenotypes, suggesting that some members of the family can substitute for others during development. In contrast, challenge of these models with pathophysiologic stimuli has elicited important roles for individual MMP enzymes (9).
Because Kim et al. used the $\alpha$ myosin heavy chain promoter, which targets expression to the cardiac myocyte predominantly postnatally, their model does not preclude an important role for an intact collagen scaffold during normal cardiac morphogenesis.

\section{Some open questions}

Well-planned and executed transgenic experiments, such as those of Kim et al., often inspire further efforts to unravel the mechanisms underlying the observed phenotype. In this case, several important questions remain regarding the regulation of cardiac ECM synthesis and turnover in this system. For example, what causes the bimodal course of collagen accumulation in this transgenic mouse? Why should overexpression of a collagenolytic enzyme increase accumulation of collagen and procollagen III mRNA at the 6-month time point, but reduce collagen levels after one year? Does a compensatory feedback loop augment collagen gene expression?

\section{MMPs must now be regarded as viable suspects in heart failure.}

Our recent experiments (7) also show a curious MMP-mediated counterregulatory phenomenon. When we produced myocardial infarction in mice deficient in MMP-9, we observed overexpression of other MMPs (7). Similarly, in the 1970s, Libby et al. (10) showed that treatment of fetal mouse hearts with a relatively specific proteinase 
inhibitor can cause overexpression of a panel of other hydrolytic enzymes, raising the possibility that the accumulation of some common substrate feeds back to regulate a variety of degradative enzymes. For example, some of the observations of Kim et al. (8) might be explained if some collagen degradation product serves as a nonspecific inducer of MMP expression.

The experiments of Kim et al. (8) suggest that the ECM must be considered together with the cardiac myocyte as one functional unit that must maintain biomechanical integrity. Cardiomyocyte hypertrophy may be an essential adaptive response to any disruption in this integrity. This scenario is astonishingly analogous to molecular studies of the touch sensation unit of Caenorhabitis elegans; the genes that allow mechanosensation encode mechanosensitive ion channels as well as unique intracellular microtubule and extracellular collagen molecules (11). Is it possible, then, that increased biomechanical stress initiated from either the inside or outside of the cardiac myocyte activates identical pathways (12)?
Finally, Kim et al. (8) propose that their transgenic mouse provides a relevant model of cardiomyopathy. Because collagen degradation is unlikely to be a usual initiating factor for this epidemic, devastating, and costly condition, this claim may meet with some skepticism. However, the MMP-1 overexpression and ECM turnover seen in this admittedly artificial system may indeed model a common step in diverse pathways leading to heart failure. Expression of several MMPs increases in ischemia, the most common cause of heart failure, as well as in dilated cardiomyopathy. It is possible that even in cardiomyopathies that arise from mutations in cytoskeletal genes, MMPs may serve as essential mediators of the dilation process. For this reason, the effects of dysregulated cardiac MMP expression may have direct bearing on the pathogenesis, and possibly the treatment, of many different types of heart failure.

1. Hunter, J.J., and Chien, K.R. 1999. Signaling pathways for cardiac hypertrophy and failure. N. Engl. J. Med. 341:1276-1283.

2. Spinale, F.G., Coker, M.L., Bond, B.R., and Zellner,
J.L. 2000. Myocardial matrix degradation and metalloproteinase activation in the failing heart: a potential therapeutic target. Cardiovasc. Res. 46:225-238.

3. Ross, R.S., et al. 1998. Beta1 integrins participate in the hypertrophic response of rat ventricular myocytes. Circ. Res. 82:1160-1172.

4. Coral-Vazquez, R., et al. 1999. Disruption of the sarcoglycan-sarcospan complex in vascular smooth muscle: a novel mechanism for cardiomyopathy and muscular dystrophy. Cell. 98:465-474.

5. Spinale, F.G., et al. 1999. Matrix metalloproteinase inhibition during the development of congestive heart failure: effects on left ventricular dimensions and function. Circ. Res. 85:364-376.

6. Rohde, L.E., et al. 1999. Matrix metalloproteinase inhibition attenuates early left ventricular enlargement after experimental myocardial infarction in mice. Circulation. 99:3063-3070.

7. Ducharme, A., et al. 2000. Targeted deletion of matrix metalloproteinase- 9 attenuates left ventricular enlargement and collagen accumulation after experimental myocardial infarction. J. Clin. Invest. 106:55-62.

8. Kim, H.E., et al. 2000. Disruption of the myocardial extracellular matrix leads to cardiac dysfunction. J. Clin. Invest. 106:857-866.

9. Werb, Z. 1997. ECM and cell surface proteolysis: regulating cellular ecology. Cell. 91:439-442.

10. Libby, P., Ingwall, J.S., and Goldberg, A.L. 1979. Reduction of protein degradation and atrophy in cultured fetal mouse hearts by leupeptin. Am.J. Physiol. 237:E35-E39.

11. Du, H., Gu, G., William, C.M., and Chalfie, M. 1996. Extracellular proteins needed for C. elegans mechanosensation. Neuron. 16:183-194.

12. Hein, S., Kostin, S., Heling, A., Maeno, Y., and Schaper, J. 2000. The role of the cytoskeleton in heart failure. Cardiovasc. Res. 45:273-278. 